\title{
Cryptococcal meningo-encephalitis
}

\author{
J. J. RIPPEY, W. A. G. ROPER, A. L. JEANES, AND M. V. BRIGHT \\ From Guy's Hospital, London
}

SYNOPSIS A case of cryptococcal meningo-encephalitis is described. Treatment with the fungicidal agent amphotericin B resulted in temporary improvement, but the patient relapsed and died 1क大 months after diagnosis. The pathology and treatment of the disease are discussed.

The incidence of meningo-encephalitis due to infection with the yeast-like organism Cryptococcus neoformans remains low in Britain but cases have been recognized with increasing frequency in recent years. The fungicidal agent amphotericin $B$ has proved to be the most effective drug yet available for the treatment of this condition, but there have so far been few published reports of its use in cases arising in this country.

We present here a clinico-pathological report of a case of cryptococcal meningo-encephalitis in a man who had never been abroad and died of the disease 17 months after diagnosis in spite of an encouraging initial response to treatment with amphotericin $\mathbf{B}$.

\section{CASE REPORT}

The patient, a man aged 60 years, was admitted to hospital in August 1962 with four weeks' history of severe and increasing frontal headache, mainly affecting the right side. There was also a history of discharge from the left ear for many years, and this had recently increased in severity. On examination he was found to be confused, disorientated, pyrexial, and obviously in severe pain. There was a profuse purulent discharge from the left ear, papilloedema mainly affecting the left eye, and diplopia on looking to the left. The left plantar reflex was extensor and there was an area of sensory impairment over the right foot. On the basis of the clinical history and physical signs, cerebral abscess secondary to chronic suppurative otitis media appeared at this stage to be the most likely diagnosis.

Radiological examination of the skull showed a densely sclerotic left mastoid process suggestive of chronic infection, but ventriculography showed no evidence of cerebral abscess. Lumbar puncture showed that the cerebrospinal fluid was under a pressure of more than $300 \mathrm{~mm}$. The fluid contained 300 red cells and 66 lymphocytes per c.mm. The protein, sugar, and chloride levels were respectively $120 \mathrm{mg}$., $34 \mathrm{mg}$., and $710 \mathrm{mg}$. per $100 \mathrm{ml}$.

Received for publication 25 November 1964.
The Lange colloidal gold reaction was 3222210000 ande the Wassermann reaction was negative.

A wet preparation of the cerebrospinal fluid depos? showed, in addition to the red and white cells, a number of yeast-like bodies, some of which were budding. Stained films showed that these organisms were Gram-positive and that they were surrounded by Gram-negative capsular material. Indian ink preparations showed the pre sence of encapsulated yeast cells with the typical appearances of Cryptococcus neoformans. The identity of the organism was subsequently confirmed by culture and a revised diagnosis of cryptococcal meningitis was now made.

FURTHER INVESTIGATIONS The haemoglobin level was $14.8 \mathrm{~g}$. per $100 \mathrm{ml}$. There were 7,000 white blood cel $\overline{\mathrm{G}}$ per c.mm., with a normal differential count, and the erythrocyte sedimentation rate was $45 \mathrm{~mm}$. in the fir hour (Westergren). Cultures of the discharge from the left ear yielded a heavy growth of a species of Proteus but were negative for Cryptococcus neoformans. $X$-ray examination of the chest showed an opacity, about $4 \mathrm{~cm}$ in diameter, situated posteriorly in the right lower lobe. Electro-encephalography showed evidence of diffuse cerebral disturbance.

MYCOLOGY The true nature of the infection first becam apparent from the characteristic appearance of the encapsulated yeast cells seen in the indian ink preparà tions of the cerebrospinal fluid (Fig. 1). The organisi grew both on blood agar and on Sabouraud's dextrose agar at room temperature and at $37^{\circ} \mathrm{C}$. Growth appeares after 48 hours' incubation and was maximal after five days, when the creamy mucoid colonies tended to coalesce

Urea was split after three days' incubation of the organism on Christensen's medium. Inoculation of serumo sugar media resulted in the production of acid, but not gas, in glucose after four days and in sucrose after 14 days. No fermentation occurred with lactose, mannito maltose, galactose, dulcite, salicin, sorbitol, adonitol, of inulin. Mice inoculated intraperitoneally with a salin suspension of the organisms died within two weeks an\& Cryptococcus neoformans was recovered from the pere toneal cavity and from the lungs. 


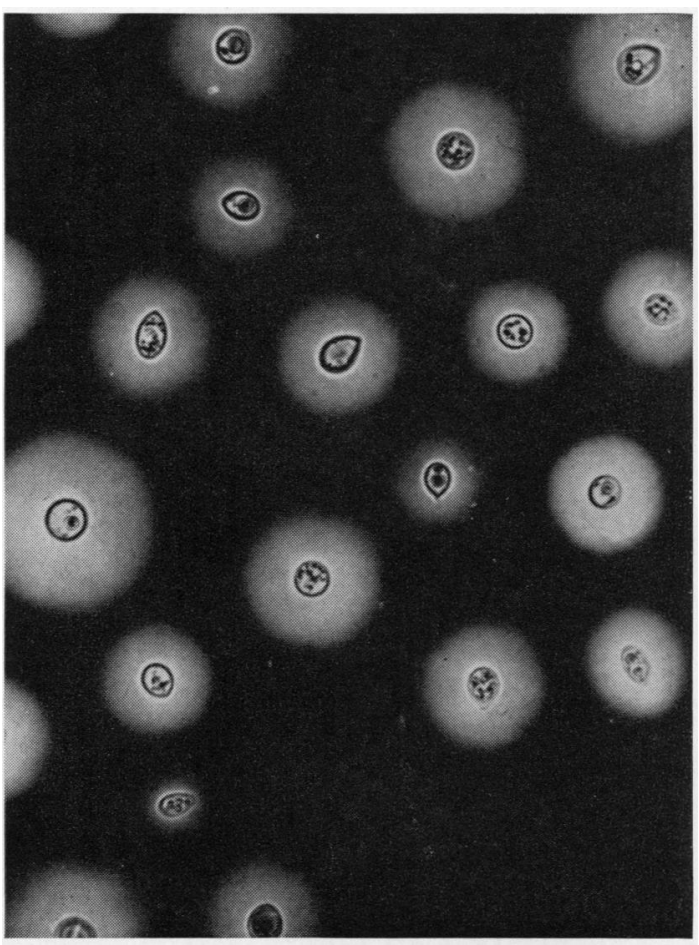

FIG. 1. Indian ink preparation of cerebrospinal fluid showing encapsulated cryptococci.

Antibiotic sensitivity tests employing impregnated paper discs on blood agar showed that the organism was resistant to all the antibiotics in routine use except colistin (Colomycin), which produced a zone of inhibition of growth $20 \mathrm{~mm}$. in diameter. Sensitivity of the organism to amphotericin B was determined by the incorporation of varying concentrations of the drug in Sabouraud's medium, both solid and liquid. In either case, it was found that growth was inhibited by a concentration of 0.25 mcg. per ml. Further tests carried out during the course of the illness showed that there was no reduction in the sensitivity of the organism to amphotericin B following the administration of this drug to the patient. A level of $0.5 \mathrm{mcg}$. per $\mathrm{ml}$. of amphotericin $\mathbf{B}$ was found in the patient's serum 15 hours after the infusion of a full dose of $1 \mathrm{mg}$. per $\mathrm{kg}$. body weight. No assayable level of the drug could be demonstrated in the cerebrospinal fluid.

Quantitative tests of colistin sensitivity were also performed, but it was found that the minimal inhibitory concentration of this antibiotic was $80 \mathrm{mcg}$. per ml., which is some four times the highest attainable blood level (McMillan, Price, MacLaren, and Scott, 1962).

Attempts were made to demonstrate antibody to Cryptococcus neoformans in the patient's serum by means of a technique based on the indirect fluorescent antibody test (Weller and Coons, 1954). Samples of serum were tested at all stages of the illness, but all gave negative results. The antigen preparations used for these tests were smears of fresh encapsulated cryptococci obtained from the peritoneal exudate of mice previously infected by intraperitoneal inoculation. The smears were treated first with the test serum and subsequently, after washing, with fluorescein-labelled rabbit anti-human globulin. When the treated preparations were examined under the fluorescence microscope, no fluorescence of the cryptococcal capsules was observed. The cryptococcal cells exhibited moderate fluorescence, but this was no brighter than the normal auto-fluorescence which is observed when untreated cryptococci are examined in this manner.

TREATMENT AND PROGRESS Specific therapy was started at the beginning of September 1962, as soon as the diagnosis became known. Amphotericin B was given as an intravenous infusion in $5 \%$ dextrose solution, lasting over six hours, on alternate days. Dosage was commenced at $0.25 \mathrm{mg}$. per $\mathrm{kg}$. body weight. In the initial stages of treatment the patient suffered severe reactions in the form of vomiting and rigors, but the full dosage of $1.0 \mathrm{mg}$. per kg. was attained after three weeks. The severity of the reactions was lessened by the incorporation of hydrocortisone sodium succinate, $100 \mathrm{mg}$., in the infusions, and by the intramuscular injection of promezathine hydrochloride, 50 mg., 30 minutes before the infusions were given.

The patient appeared to respond well to treatment. His temperature became normal after one week, and he became free from headache after two weeks. During this period, however, he developed a severe right-sided foot drop, although there was some improvement in the sensation over this foot. The changes which took place in the cerebrospinal fluid during treatment are summarized in Table I. The cell count and protein level remained abnormal, but there was a gradual diminution in the number of yeast cells present, and although these never disappeared completely from the fluid, cultures became sterile after two weeks.

A total of $2.2 \mathrm{~g}$. of amphotericin B was given over three months and at the end of this time the patient appeared to be well both mentally and physically except for persistence of the foot drop. The blood urea level, which had risen to $64 \mathrm{mg}$. per $100 \mathrm{ml}$. during treatment, became normal again within a few days after this was discontinued.

In December 1962 a left radical mastoidectomy was performed by Mr. L. F. W. Salmon, and the patient was discharged from hospital later in the same month. Histological examination of material removed at mastoidectomy showed the presence of non-specific inflammatory granulation tissue, but no cryptococci were seen in sections stained either with haematoxylin and eosin or by the periodic acid-Schiff technique.

During the next few months the patient's condition showed little change, but it was noteworthy that further radiographs of the chest showed a considerable diminution in the size of the opacity in the right lung. In May 1963, however, cultures of the cerebrospinal fluid again became positive for Cryptococcus neoformans. He was readmitted for a further course of amphotericin B, of which a total dosage of $840 \mathrm{mg}$. was given during the next four weeks. Cultures remained positive, but he was extremely reluctant to undergo any further treatment and therefore was allowed to return home. 
TABLE I

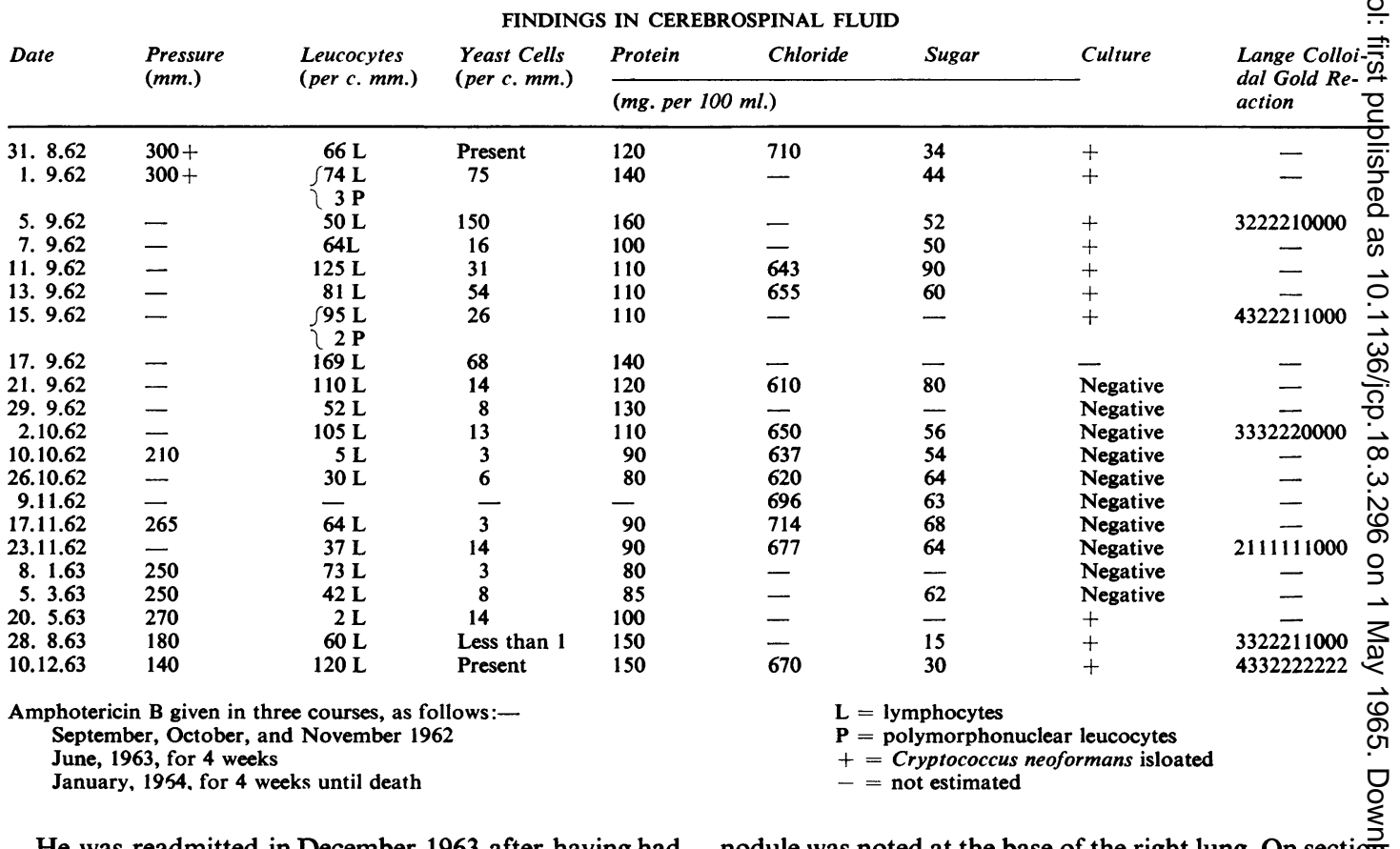

He was readmitted in December 1963 after having had a series of falls, and on this occasion he was found to be disorientated and confused, with hemiparesis involving the left side. The cerebrospinal fluid now contained many cryptococcal cells, and cultures were still positive. Carotid angiography showed the presence of hydrocephalus due to a right temporo-thalamic space-occupying lesion. A third course of amphotericin B was therefore instituted, but the patient's condition deteriorated and he died in February 1964 after having received $850 \mathrm{mg}$. of the drug over the previous four weeks.

NECROPSY There was intense congestion of both lungs, but no oedema or bronchopneumonia. A pale, projecting

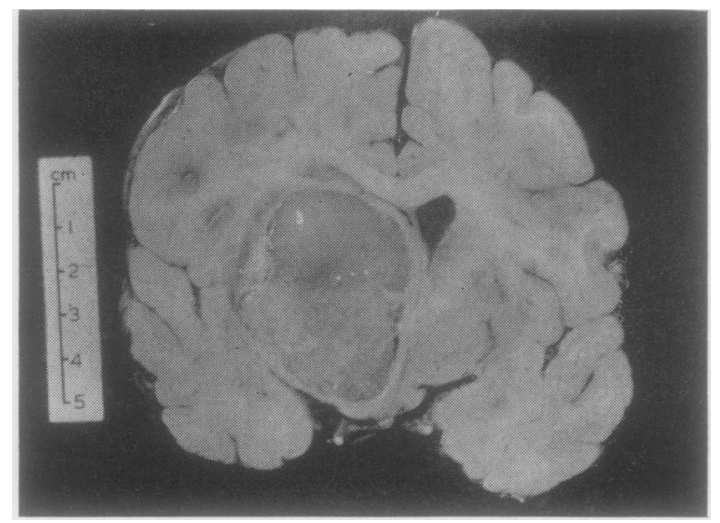

FIG. 2. Macroscopic section of brain showing lesion in the region of the right basal ganglia. nodule was noted at the base of the right lung. On sectiof, a triangular wedge of consolidation, measuring $0.8 \mathrm{~cm}$. $2 \mathrm{~cm}$., was found beneath the pleura. The lesion consistid of firm, pale grey tissue, with a glistening mucoid appearance, projecting from the cut surface of the lung.을

The brain was a little rounded and swollen, but the meninges were not grossly abnormal. A sharply dema cated, expanding lesion, composed of mucoid and gefitinous tissue and measuring $5 \mathrm{~cm} . \times 3 \mathrm{~cm}$., occupied the region of the right basal ganglia, displacing the latefal ventricle medially and the putamen and globus pallidits laterally (Fig. 2). A small seedling was present in the cortex of the right occipital lobe and two others of the same size were found in the cerebellum. A mucoid nodufe of pinhead size was seen in the vermis, and a grey, ifdefined area was noted in the white matter of the left orbito-frontal region. The leptomeninges of the spingl cord were not unduly thickened, apart from sorñ doubtful thickening in the ventral fissure.

HISTOLOGY The wedge-shaped lesion in the lung showed encapsulated cryptococci lying in cyst-like spaces in dense fibrous scar tissue which was infiltrated by monanuclear cells. The surrounding lung parenchyma showod an interstitial fibrosis. The organisms were Granpositive and P.A.S.-positive and their capsules stained intensely with mucicarmine.

The main lesion in the brain showed the presence of masses of cryptococci lying in a poorly staining matr The surrounding brain tissue was compressed into 置 narrow rim, and there was very little cellular reaction. The brain tissue also contained numerous corposa amylacea which were distinguished from the cryptocoggi 
by their poor staining with mucicarmine. A few cryptococci and multinucleate giant cells could be seen in the cerebral subarachnoid space. The spinal meninges showed thickening and infiltration by mononuclear cells.

\section{DISCUSSION}

The clinical and pathological features of cryptococcal infection and the characteristics of the causative organism are well described by Conant, Smith, Baker, Callaway, and Martin (1954). Extensive reviews have been published by Cox and Tolhurst (1946) and Littman and Zimmerman (1956) respectively in Australia and the United States of America, in which countries the disease occurs more commonly than in Britain. A brief review of the British cases reported up to 1958 , together with a description of three further cases, was published in that year by Rose, Grant, and Jeanes. More recently, McMath and Hussain (1961) have described a case of the disease in a 4-year-old boy who died within a few hours of the commencement of amphotericin B therapy, after having been ill for 16 days.

Cryptococcal infection, which is also known as torulosis or European blastomycosis, commonly presents itself in the form of chronic meningoencephalitis. There is frequently an associated pulmonary lesion, and the skin or other parts of the body may sometimes be involved. The cerebrospinal fluid usually shows an increased lymphocyte count, the protein level is raised, the sugar and chloride levels are reduced, and the Lange colloidal gold reaction may be paretic or luetic in type. The principal diagnostic feature is the presence in the fluid of yeast-like bodies which in indian ink preparations are seen to possess large transparent capsules. The immune response of infected patients to Cryptococcus neoformans is characteristically poor, and there is usually remarkably little cellular reaction to the presence of cryptococci in the tissues. If untreated, the disease usually progresses to a fatal outcome within a few weeks or months, but occasionally pursues a much longer course.

The case reported here was typical in many respects, but the onset was complicated by the presence of non-specific chronic suppurative otitis media which led to a suspected clinical diagnosis of otogenic cerebral abscess. This diagnosis was excluded by ventriculography, however, and the true nature of the disease was revealed by the subsequent finding of cryptococci in the cerebrospinal fluid. It is likely that the ear infection was purely incidental and in no way related to the cerebrospinal infection, since repeated cultures of the aural discharge were negative for Cryptococcus neoformans, and histological examination of the tissue removed at mas- toidectomy showed no evidence of cryptococcal infection.

The clinical course of the illness, the cerebrospinal fluid findings and the pathology of the lesions in the brain and lung were typical of cryptococcosis and need no further elaboration here.

Because of the characteristic lack of anti-cryptococcal activity in the serum of patients with this disease, there are no standard immunological tests which may be used in the diagnosis. A promising development was reported by Vogel and Padula (1958), who by means of the indirect fluorescent antibody test were able to detect specific antibody, though to a titre of only $1: 4$, in the serum of a patient with systemic cryptococcal infection. In our case, however, this test gave consistently negative results throughout the entire course of the illness, although the same method has been used with success for the detection of antibodies to other pathogenic yeasts (Jeanes, 1964).

Following the introduction of amphotericin B, there have been a number of reports of successfully treated cases of cryptococcal infection, but in view of the chronicity of the disease and the tendency to relapse, it is perhaps too early to judge the ability of the drug to achieve total eradication of the infection. In a series of $\mathbf{4 0}$ patients reported by Butler, Alling, Spickard, and Utz (1964) five died despite treatment with amphotericin B and a further 11 relapsed after having shown initial improvement. There is no doubt, however, that amphotericin B is the most effective drug yet available. This substance is poorly absorbed when given by mouth, and the usual method of administration is by slow intravenous infusion. Because of possible toxic manifestations, which include headache, nausea, vomiting, fever, rigors, and a rise in blood urea nitrogen, it is necessary to proceed to full dosage gradually and with caution, and to control therapy with repeated tests of hepatic and renal function. The drug may also be injected intrathecally, but this method of administration is not without the risk of complications such as pain, paresis, chemical meningitis, or secondary infection of the meninges (Spickard, Butler, Andriole, and Utz, 1963).

In this case, amphotericin B therapy may be said to have been partially effective. The patient was extremely ill when first admitted to hospital but made a good clinical recovery following his first course of treatment. He survived for longer than would be expected from the natural history of the disease (Conant et al., 1954), although cases of up to 16 years' duration have been recorded (Beeson, 1952). It is also of interest that the pulmonary lesion showed a considerable reduction in size following treatment. 
In spite of the apparent response to the first course of treatment, however, the cerebrospinal fluid never became completely free from cryptococci although cultures were sterile over a long period. This suggests that the amphotericin $B$ was reaching the fluid in fungistatic rather than fungicidal concentrations, and it is possible that the additional administration of the drug by the intrathecal route would have been more effective. This was withheld because the patient was already experiencing severe reactions following the intravenous infusions and because it was felt unlikely that he would tolerate intrathecal therapy in addition.

Cryptococcal meningo-encephalitis is obviously a formidable therapeutic challenge. Amphotericin B remains at present the most effective agent with which to meet this challenge, but presumably it is most likely to succeed when diagnosis is made early. Since the incidence of the disease in Britain appears to be increasing, it is suggested that this diagnosis be considered in any case of meningitis for which no other explanation can be found.

We wish to thank Dr. C. Hardwick for permission to publish this case. We also thank the other members of the staff of Guy's Hospital who were concerned, partic- ularly Mr. Murray A. Falconer, whose advice wæ3 sought on the neurosurgical aspects of the case, and $D$ W. H. H. Merivale, in whose department most of the laboratory investigations were performed.

We are also grateful to Dr. J. B. Cavanagh and Dr. $\$^{5}$ Leibowitz for their report on the necropsy findings Mr. F. J. Gibbs for his technical assistance, and Messro E. R. Squibb and Sons for information on their produef amphotericin B (Fungizone).

\section{REFERENCES}

Beeson, P. B. (1952). Arch. intern. Med., 89, 797.

Butler, W. T., Alling, D. W., Spickard, A., and Utz, J. P. (1964) New Engl.J. Med., 270, 59.

Conant, N. F., Smith, D. T., Baker, R. D., Callaway, J. L., añ Martin, D. S. (1954). Manual of Clinical Mycology, p. 149 Saunders, Philadelphia.

Cox, L. B., and Tolhurst, J. C. (1946). Human Torulosis. Melbour University, Melbourne.

Jeanes, A. L. (1964). Guys Hosp. Rep., 113, 136.

Littman, M. L., and Zimmerman, L. E. (1956). Cryptococcosis. Grunte and Stratton, New York.

McMath, W. F. T., and Hussain, K. K. (1961). Brit. med. J., 2, 91. -

McMillan, M., Price, T. M. L., MacLaren, D. M., and Scott, G. (1962). Lancet, $2,737$.

Rose, F. C., Grant, Helen C., and Jeanes, A. L. (1958). Brain, 81, 542.

Spickard, A., Butler, W. T., Andriole, V., and Utz, J. P. (1963). Anचु intern. Med., 58, 66.

Vogel, R. A., and Padula, J. F. (1958). Proc. Soc. exp. Biol. (N.YG] 98,135 .

Weller, T. H., and Coons, A. H. (1954). Ibid., 86, 789. 